\title{
A NOTE ON TWO POINT BOUNDARY PROBLEMS FOR NONLINEAR MATRIX DIFFERENTIAL SYSTEMS
}

\author{
GARRET J. ETGEN
}

\begin{abstract}
This note is concerned with second order, nonlinear matrix differential systems involving a parameter together with boundary conditions specified at two points. The objective is to establish sufficient conditions for the existence of eigenvalues for the system. The results presented here represent extensions of recent work of the author. The principal motivation is provided by W. M. Whyburn's work on nonlinear boundary problems for second order differential systems.
\end{abstract}

1. Introduction. This note is concerned with the system of $2 n^{2}$ firstorder, nonlinear differential equations:

$$
\begin{aligned}
& y_{i j}^{\prime}=\sum_{h=1}^{n} k_{i h}\left[x, y_{11}, y_{12}, \cdots, y_{n n}, z_{11}, z_{12}, \cdots, z_{n n} ; \lambda\right] z_{h j}, \\
& z_{i j}^{\prime}=\sum_{h=1}^{n}-g_{i h}\left[x, y_{11}, y_{12}, \cdots, y_{n n}, z_{11}, z_{12}, \cdots, z_{n n} ; \lambda\right] y_{h j},
\end{aligned}
$$

$1 \leqq i, j \leqq n$, defined on $X: a \leqq x \leqq b, L: \lambda_{0}-\delta<\lambda<\lambda_{0}+\delta, 0<$ $\delta \leqq \infty$. Nonlinear systems of this form were introduced in [2] and have been represented in the matrix form

$$
Y^{\prime}=K(x ; Y ; Z ; \lambda) Z, \quad Z^{\prime}=-G(x ; Y ; Z ; \lambda) Y .
$$

Studies concerned with the oscillation of solutions of nonlinear matrix differential equations have been conducted by H. C. Howard [5], E. C. Tomastik [6] and the author [2], [3]. In addition to oscillation, [2] also contains some results establishing the existence of eigenvalues for certain simple two point boundary problems. The purpose of this note is to extend the results presented in [4], where the linear version of (1) is studied, to the nonlinear case, thereby improving the work in [2].

2. The two point boundary problem. We consider (1) together with the two point boundary conditions

$$
\begin{aligned}
A(\lambda) Y(a, \lambda)-B(\lambda) Z(a, \lambda) & =0, \\
\operatorname{det}[C(\lambda) Y(b, \lambda)-D(\lambda) Z(b, \lambda)] & =0,
\end{aligned}
$$

Received by the editors February 8, 1971 and, in revised form, April 9, 1971.

AMS 1970 Subject classifications. Primary 34B15.

Key words and phrases. Nonlinear matrix differential equations, two point boundary problems, existence of eigenvalues.

()American Mathematical Society 1972 
where $A, B, C$ and $D$ are $n \times n$ matrices of continuous real-valued functions on $L$. We assume, specifically, that the coefficients in the problem satisfy the following hypotheses:

$\mathrm{H}_{1}$. The functions $k_{i j}$ and $g_{i j}$ are real-valued and satisfy conditions insuring the existence of a solution when appropriate initial conditions are specified.

$\mathrm{H}_{2}$. Each of $K$ and $G$ is symmetric on $X L$ for all pairs $Y, Z$.

$\mathrm{H}_{3} . K$ is positive definite on $X L$ for all pairs $Y, Z$.

$\mathrm{H}_{4} \cdot A^{*}(\lambda) B(\lambda) \equiv B^{*}(\lambda) A(\lambda), C^{*}(\lambda) D(\lambda) \equiv D^{*}(\lambda) C(\lambda)$ on $L$.

$\mathrm{H}_{5}$. det $[B(\lambda)] \neq 0$ and $\operatorname{det}\left[C(\lambda) B^{*}(\lambda)-D(\lambda) A^{*}(\lambda)\right] \neq 0$ on $L$.

$\mathrm{H}_{6} . A^{*}(\lambda) A(\lambda)+B^{*}(\lambda) B(\lambda) \equiv C^{*}(\lambda) C(\lambda)+D^{*}(\lambda) D(\lambda) \equiv I$ on $L$.

(* denotes transpose and $I$ the identity matrix.)

A solution pair $\{Y(x, \lambda), Z(x, \lambda)\}$ is called nontrivial if $Y^{*} Y+Z^{*} Z$ is positive definite on $X$ for each $\lambda$ on $L$. We seek to establish the existence of values of $\lambda$ which correspond to a nontrivial solution pair of $\{Y(x, \lambda)$, $Z(x, \lambda)\}$ of (1) satisfying (2). Such values of $\lambda$ are called the eigenvalues of the system.

If $\{Y(x, \lambda), Z(x, \lambda)\}$ is a solution pair of (1) satisfying

$$
Y(a, \lambda) \equiv B^{*}(\lambda), \quad Z(a, \lambda) \equiv A^{*}(\lambda) \quad \text { on } \quad L,
$$

then, as in the linear case (see e.g. [4]), hypotheses $\mathrm{H}_{4}$ and $\mathrm{H}_{6}$ imply that the pair is nontrivial and conjoined, i.e., $Y^{*} Z \equiv Z^{*} Y$ on $X$ for each $\lambda$ on $L$.

3. Existence of eigenvalues. In establishing the existence of eigenvalues for the system (1), (2), we make extensive use of the corresponding results which were derived for the linear case [4].

THEOREM 1. Let $\{Y(x, \lambda), Z(x, \lambda)\}$ be a solution of $(1),(3)$. Let $\Omega(x, \lambda)$ and $q(\lambda)$ be defined by

$$
\Omega(x, \lambda)=Z^{*} K(x ; Y ; Z ; \lambda) Z+Y^{*} G(x, Y ; Z ; \lambda) Y,
$$

and

$$
q(x, \lambda)=2 \int_{a}^{x} \operatorname{tr} \Omega(t, \lambda) d t,
$$

respectively. Then for each $x$ on $X, q(x, \lambda)>-2 n \pi$ on $L$.

Proof. As noted above, a solution pair $\{Y(x, \lambda), Z(x, \lambda)\}$ of (1), (3) is nontrivial and conjoined. It now follows that the matrix $\Psi(x, \lambda)$ defined by

$$
\Psi(x, \lambda)=(Z+i Y)(Z-i Y)^{-1}
$$

exists and is unitary on $X$ for each $\lambda$ on $L$. Also, for each fixed $\lambda, \Psi$ 
satisfies the first order differential

$$
\Psi^{\prime}=2 i \Psi \Omega
$$

where $\Omega$ is the Hermitian matrix given by (4). Let $\psi_{j}(x, \lambda), j=1,2, \cdots, n$, denote the characteristic roots of $\Psi$ and $\omega_{j}(x, \lambda), j=1,2, \cdots, n$, their respective arguments, where it is assumed that $0 \leqq \omega_{j}\left(a, \lambda_{0}\right)<2 \pi, j=$ $1,2, \cdots, n$, and that the functions $\omega_{j}(x, \lambda)$ are continued as continuous functions on $X L$. For each fixed $\lambda, y^{\prime}{ }_{j}(x, \lambda)=+1$ for at least one $j$ if and only if det $Y(x, \lambda)=0$, the number of $\psi_{j}(x, \lambda)$ having the value +1 equals the multiplicity of the zero of $\operatorname{det} Y(x, \lambda)$, the functions $\psi_{j}(x, \lambda)$ move positively on the unit circle at the point +1 , and

$$
q(x, \lambda)=2 \int_{a}^{x} \operatorname{tr} \Omega(t, \lambda) d t=\sum_{j=1}^{n}\left[\omega_{j}(x, \lambda)-\omega_{j}(a, \lambda)\right] .
$$

The assumption $\mathrm{H}_{5}$ implies $0<\omega_{j}(a, \lambda)<2 \pi$ on $L$ for $j=1,2, \cdots, n$. Since the characteristic roots $\omega_{j}(x, \lambda)$ move positively at the point +1 for each fixed $\lambda$, we have $\omega_{j}(x, \lambda)>0$ on $X, j=1,2, \cdots, n$. Thus

$$
q(x, \lambda)=\sum_{j=1}^{n}\left[\omega_{j}(x, \lambda)-\omega_{j}(a, \lambda)\right]>-\sum_{j=1}^{n} \omega_{j}(a, \lambda)>-2 n \pi
$$

on $L$ for each $x$ on $X$.

Our next theorem establishes a sufficient condition for the existence of eigenvalues for (1), (2).

THEOREM 2. Let $\{Y(x, \lambda), Z(x, \lambda)\}$ be a solution of (1), (3) and let $\Omega(x, \lambda)$ and $q(x, \lambda)$ be defined by (4) and (5). Let $h$ be an integer such that g.l.b. $q(\lambda) \leqq 2 h \pi$ and let $m$ be an integer such that l.u.b. $q(\lambda) \geqq 2 m n \pi$.

If $m>h+n$ with $k$ the largest nonnegative integer such that $m-(h+n)$ $\geqq k n$, then there exist at least $k$ nonempty sets of eigenvalues $J_{0}, J_{1}, \cdots$, $J_{k-1}$ for the system (1), (2). If $m$ may be chosen arbitrarily large, then there exist infinitely many nonempty sets of eigenvalues $J_{0}, J_{1}, \cdots$ for the system (1), (2). In either case, the sets of eigenvalues $J_{0}, J_{1}, \cdots$ may be chosen so that if $\lambda_{p} \in J_{p}, p \geqq 1$, then the corresponding solution $\left\{Y\left(x, \lambda_{p}\right), Z\left(x, \lambda_{p}\right)\right\}$ has the property that det $Y\left(x, \lambda_{p}^{*}\right)$ has at least $p-1$ zeros on $X$, multiple zeros being counted according to their multiplicities.

Proof. Define the pair of matrices $\{U(x, \lambda), V(x, \lambda)\}$ on $X L$ by

$$
\begin{aligned}
& U(x, \lambda)=C(\lambda) Y(x, \lambda)-D(\lambda) Z(x, \lambda), \\
& V(x, \lambda)=C(\lambda) Z(x, \lambda)+D(\lambda) Y(x, \lambda) .
\end{aligned}
$$

It is readily verified using the properties of the pairs $\{Y, Z\}$ and $\{C, D\}$ that the pair $\{U, V\}$ has the properties: $U^{*} U+V^{*} V$ is positive definite and $U^{*} V=V^{*} U$ on $X$ for each $\lambda$ on $L$. The eigenvalues for the system 
(1), (2) are the values of $\lambda$ for which $\operatorname{det} U(b, \lambda)=0$. As for solution pairs of (1), (3), it is readily verified that the matrix $\Phi(x, \lambda)$ defined by

$$
\Phi(x, \lambda)=(V+i U)(V-i U)^{-1}
$$

exists and is unitary on $X$ for each $\lambda$ on $L$. For each fixed $\lambda$ on $L, \Phi$ satisfies the first order differential equation

$$
\Phi^{\prime}=2 i \Phi \bar{\Omega}
$$

where $\bar{\Omega}$ is the Hermitian matrix

$$
\bar{\Omega}=\left(C^{*}-i D^{*}\right)^{-1} \Omega(C+i D)^{-1}=(C+i D) \Omega(C+i D)^{-1} .
$$

Letting $\varphi_{j}(x, \lambda), j=1,2, \cdots, n$, denote the characteristic roots of $\Phi$ with $\beta_{j}(x, \lambda), j=1,2, \cdots, n$, their respective arguments, where we again assume $0 \leqq \beta_{j}\left(a, \lambda_{0}\right)<2 \pi$ and each $\beta_{j}(x, \lambda)$ is continued as a continuous function on $X L$, we have, for each $\lambda, \varphi_{j}(x, \lambda)=+1$ for at least one $j$ if and only if det $U(x, \lambda)=0$. We also have, since $\bar{\Omega}$ is similar to $\Omega$,

$$
\begin{aligned}
\sum_{j=1}^{n}\left[\beta_{j}(x, \lambda)-\beta_{j}(a, \lambda)\right] & =2 \int_{a}^{x} \operatorname{tr} \bar{\Omega}(t, \lambda) d t=2 \int_{a}^{x} \operatorname{tr} \Omega(t, \lambda) d t \\
& =q(x, \lambda)=\sum_{j=1}^{n}\left[\omega_{j}(x, \lambda)-\omega_{j}(a, \lambda)\right]
\end{aligned}
$$

for each $\lambda$ on $L$. In particular, in view of $\mathrm{H}_{5}, 0<\beta_{j}(a, \lambda)<2 \pi, 1 \leqq j \leqq n$, and so for $x=b$, we obtain, from (13),

$$
q(b, \lambda)<\sum_{j=1}^{n} \beta_{j}(b, \lambda)<q(b, \lambda)+2 n \pi .
$$

The remainder of the proof of the theorem can now be accomplished exactly as in the linear case. Specifically, let $\beta(\lambda)=\sum_{j=1}^{n} \beta_{j}(b, \lambda)$ and let $h, k$ and $m$ be the integers with the properties described in the hypothesis. Now, there exists a point $\bar{\lambda}$ on $L$ such that $q(b, \bar{\lambda}) \leqq 2 h \pi$ and there exists a point $\lambda^{*}$ on $L$ such that $q\left(b, \lambda^{*}\right) \geqq 2 m n \pi$ with $m-(h+n) \geqq$ $k \cdot n$. We assume $\bar{\lambda}<\lambda^{*}$. From (14) we have $\beta(\bar{\lambda}) \leqq 2(h+n) \pi$ and $\beta\left(\lambda^{*}\right) \geqq 2 m n \pi$. By the continuity of $\beta(\lambda)$ on $L$, there exist $k+1$ values of $\lambda, \lambda_{0}, \lambda_{1}, \cdots, \lambda_{k}$, with $\bar{\lambda} \leqq \lambda_{0}<\lambda_{1}<\cdots<\lambda_{k} \leqq \lambda^{*}$, such that $\beta\left(\lambda_{p}\right)=$ $2(h+n+p n) \pi, p=0,1, \cdots, k$. Now as $\lambda$ increases from $\lambda_{p}$ to $\lambda_{p+1}$, $0 \leqq p \leqq k-1, \beta(\lambda)$ increases by $2 n \pi$ and, consequently, at least one of the functions $\beta_{j}(b, \lambda)$ must increase by at least $2 \pi$ so that $\beta_{j}(b, \lambda) \equiv 0$ $(\bmod 2 \pi)$ for some value of $\lambda$ on the interval $\left[\lambda_{p}, \lambda_{p+1}\right]$. We conclude, therefore, that det $U(b, \lambda)=0$ for at least one value of $\lambda$ on $\left[\lambda_{p}, \lambda_{p+1}\right]$ and the first part of the theorem follows. The remaining portions of the theorem are established exactly as in [4, Corollaries 2,3$]$. 
We note that this theorem substantially improves the results in $[2, \S 3]$ where very special two point boundary problems were considered and where it was required that each of the symmetric matrices $K$ and $G$ be positive definite on $X L$ for all absolutely continuous pairs $\{Y, Z\}$. We note also that this theorem has a drawback not encountered in the linear case, namely, the conditions insuring the existence of eigenvalues depend upon the choice of a solution pair $\{Y, Z\}$ of (1), (3). To overcome this drawback we proceed as follows: Let $\Gamma$ denote the collection to which the ordered pair $\{Y, Z\}$ belongs if and only if each of $Y$ and $Z$ is an $n \times n$ absolutely continuous matrix on $X L, Y^{*} Z \equiv Z^{*} Y$ and $Y^{*} Y+Z^{*} Z$ is positive definite on $X$ for each $\lambda$ on $L$. Define the $n \times n$ symmetric matrices $S$ and $T$ on $X \Gamma L$ by

$$
\begin{aligned}
S(x ; Y ; Z ; \lambda)= & K(x ; Y ; Z ; \lambda)+G(x ; Y ; Z ; \lambda) \\
& -[K(x ; Y ; Z ; \lambda)-G(X ; Y ; Z ; \lambda)], \\
T(x ; Y ; Z ; \lambda)= & K(x ; Y ; Z ; \lambda)+G(x ; Y ; Z ; \lambda) \\
& +[K(x ; Y ; Z ; \lambda)-G(x ; Y ; Z ; \lambda)],
\end{aligned}
$$

where $[M]$ denotes the nonnegative definite square root of $M^{*} M$. Extending the inequalities established by Atkinson [1, Chapter 10], we have

$$
\operatorname{tr} S \leqq 2 \operatorname{tr} \Omega(x, \lambda) \leqq \operatorname{tr} T
$$

whenever $\{Y, Z\}$ is a nontrivial conjoined solution of (1). For each $\lambda$ on $L$, define $u(\lambda)$ and $v(\lambda)$ by

$$
u(\lambda)=\text { g.l.b. } \operatorname{tr} S \text { on } X \Gamma, \quad v(\lambda)=\text { l.u.b. } \operatorname{tr} T \text { on } X \Gamma .
$$

THEOREM 3. If there exist integers $h$ and $m$ with the properties

(i) $v(\bar{\lambda})(b-a)<2 h \pi$ for some $\bar{\lambda}$ on $L$,

(ii) $u\left(\lambda^{*}\right)(b-a)>2 m n \pi$ for some $\lambda^{*}$ on $L$, and

(iii) $m>h+n$ with $k$ the largest nonnegative integer such that

$$
m-(h+n)>k n,
$$

then there exists at least $k$ nonempty sets of eigenvalues $H_{0}, H_{1}, \cdots, H_{k-1}$ for the system (1), (2). If the integer $m$ may be chosen arbitrarily large, then there exist infinitely many nonempty sets of eigenvalues for the system.

Proof. Let $\{Y(x, \lambda), Z(x, \lambda)\}$ be any solution of (1), (3). Using the matrices defined in the proof of Theorem 1 and proceeding as in that proof, we have

$$
\begin{aligned}
v(\lambda)(b-a) & \geqq 2 \int_{a}^{b} \operatorname{tr} \Omega(x, \lambda) d x=\sum_{j=1}^{n}\left[w_{j}(b, \lambda)-w_{j}(a, \lambda)\right] \\
& =\sum_{j=1}^{n}\left[\beta_{j}(b, \lambda)-\beta_{j}(a, \lambda)\right]>-2 n \pi .
\end{aligned}
$$


Thus, for $\lambda=\bar{\lambda}$,

$$
-2 n \pi<2 \int_{a}^{b} \operatorname{tr} \Omega(x, \bar{\lambda}) d x<2 h \pi .
$$

Similarly, for $\lambda=\lambda^{*}, u\left(\lambda^{*}\right)>2 m n \pi$ implies $2 \int_{a}^{b} \operatorname{tr} \Omega\left(x, \lambda^{*}\right) d x>2 m n \pi$. Clearly, $\bar{\lambda} \neq \lambda^{*}$ since $\operatorname{tr} S \leqq \operatorname{tr} T$ on $X \Gamma L$. The proof is now completed exactly as in Theorem 2 .

\section{REFERENCES}

1. F. V. Atkinson, Discrete and continuous boundary problems, Math. in Science and Engineering, vol. 8, Academic Press, New York, 1964. MR 31 \#416.

2. G. J. Etgen, Oscillatory properties of certain nonlinear matrix differential systems of second order, Trans. Amer. Math. Soc. 122 (1966), 289-310. MR 32 \#7834.

3. - Oscillation criteria for nonlinear second order matrix differential equations, Proc. Amer. Math. Soc. 27 (1971), 259-267.

4. - Two-point boundary problems for second order matrix differential equations, Trans. Amer. Math. Soc. 149 (1970), 119-132.

5. H. C. Howard, Oscillation criteria for matrix differential equations, Canad. J. Math. 19 (1967), 184-199. MR 353126.

6. E. C. Tomastik, Oscillation of nonlinear matrix differential equations of second order, Proc. Amer. Math. Soc. 19 (1968), 1427-1431. MR 38 \#372.

7. W. M. Whyburn, Existence and oscillation theorems for non-linear differential equations of second order, Trans. Amer. Math. Soc. 30 (1928), 848-854.

8. - A nonlinear boundary value problem for second order differential systems, Pacific J. Math. 5 (1955), 147-160. MR 16, 1027.

Department of Mathematics, University of Houston, Houston, Texas 77004 